\title{
Disease-mediated piglet mortality prevents wild boar population growth in fenced overabundant settings
}

\begin{abstract}
Assessing Eurasian wild boar (Sus scrofa) mortality is a key for understanding population dynamics and adjusting hunting harvest and population management. We used radio-tagging and video-trapping to quantify piglet summer mortality in a managed (i.e. fenced and year-round fed) wild boar population from southern Spain. We used two independent tools to assess wild boar mortality, i.e. radio-tracking and video footage. A total of 32 wild boar piglets were captured at selective feeders acting as cage-traps, and all the piglets were equipped with ear-tag transmitters including a mortality sensor. Additionally, 20 camera traps were set up in video mode at wild boar feeders. Videos were visualized calculating the proportion of piglets seen per adult as a second indicator of piglet mortality. The survival analysis of radio-tagged individuals indicated an average survival of $48 \%$ after 180 days of capturing. Mortality was particularly pronounced in the first 2 months, i.e. during summer. Video footage evidenced a progressive decline in the piglet-to-adult ratio throughout summer. Between June and September, this ratio declined by $80.5 \%$. Dead piglets were in poor body condition, and respiratory lesions compatible with the porcine respiratory disease complex (PRDC) were observed in $86 \%$ of them. Porcine circovirus type 2 (PCV2) was the most prevalent pathogen $(61 \%)$ in this study, and its prevalence showed an increasing trend depending on the lung lesion score. We postulate that high piglet mortality at early age might represent a disease-mediated density-dependent mechanism limiting wild boar population growth in semi-intensive management regimes, characterized by year-round feeding and fencing.
\end{abstract}

